\title{
Splitting an Uncertain (Natural) Capital *
}

\author{
Jérémy Laurent-Lucchetti, ${ }^{\dagger}$ Justin Leroux ${ }^{\ddagger}$ \\ and Bernard Sinclair-Desgagné ${ }^{\S}$
}

February 1, 2011

\footnotetext{
${ }^{*}$ We are grateful for stimulating conversations with Olivier Bochet, Joseph Harrington, Michael McBride, Hervé Moulin, Nicolas Sahuguet, Maher Said, Carlos Seiglie and Gunter Stefan. We also acknowledge valuable feedback from participants to the Montréal Natural Resource and Environmental Economics Workshop, the Montréal Economic Theory Group, the Société canadienne de sciences économiques 2010 conference, the 2010 World Congress of Environmental and Resource Economists, the 2011 American Economic Association Meeting, and seminars at the University of Bern, Humboldt University, the European School of Management and Technology in Berlin, and the University of Norwich, as well as Financial support from the Social Sciences and Humanities Research Council of Canada (SSHRC).

${ }^{\dagger}$ Department of Economics and Oeschger Center for Climate Change Research, University of Bern.

${ }^{\ddagger}$ HEC Montréal, CIRANO and CIRPÉE.

${ }^{\S}$ HEC Montréal, CIRANO, and École polytechnique de Paris.
} 


\begin{abstract}
Most natural commons are subject to discontinuities and threshold effects, so their gradual depletion may result in a sudden irreversible loss of the associated ecological services. Yet, it is often impossible to locate these thresholds with certainty. We analyze this context using a variant of the divide-the-dollar game, in which the amount to be split among players follows a discrete or multimodal probability distribution. 'Cautious equilibria' - where agents collectively behave as if the worst-case scenario were certain - are found to coexist with 'dangerous equilibria' - where overall demand for ecological services might lead to their collapse - and 'dreadful equilibria' - where agents collectively request so much natural capital that a collapse of ecological services is certain, even if all agents are risk averse. Communication/cooperation among agents, however, which raises the possibility of coordinated group deviations, would eliminate dreadful equilibria and reduce the occurrence of dangerous equilibria, while cautious equilibria are robust to such deviations. A direct corollary is that dangerous equilibria are Pareto-dominated by any cautious equilibrium in which all agents claim less natural capital. These results shed light on the management of common-pool resources, international climate change negotiations, and the implementation of precautionary policies.
\end{abstract}

Keywords: Common-pool resources, Ecological thresholds, Dividethe-dollar game, Coalition-proof Nash equilibrium

JEL Classification: Q50, C72, D74 


\section{Introduction}

Human societies rely on a number of indispensable ecological services, such as water purification, livestock support, flood prevention, waste recycling, climate stability, erosion avoidance and fresh air, for their economic activities and wellbeing. These services are provided by forests, lakes, coral reefs, savannas, wetlands, oceans, the troposphere and other ecosystems which typically are 'common-pool resources,' i.e. resources "(...) from which it is difficult to exclude or limit users once the resource is provided, and one person's consumption of resource units makes those units unavailable to others" (Ostrom 1999, p. 497). The impossibility to exclude potential beneficiaries while there are inevitable capacity constraints (at least in the short run) makes such ecosystems, and the corresponding ecological services, particularly prone to a 'tragedy of the commons.'

Over the last decades, significant research efforts have considered how human societies can, and actually do, cope with this problem. ${ }^{1}$ Taking stock of this literature, this paper now builds on two well-documented additional observations. First, the provision of ecological services is subject to discontinuities, bifurcations or threshold effects that may show up rather abruptly, following persistent abuses of the involved ecosystems (Scheffer et al. 2001). In agriculture, for example, it is often the case that "(...) no perceptible change in the environmental state occurs unless a specified farming practice is applied with a minimal intensity and on a minimal area in the zone of interest" (Dupraz et al. 2009, p. 613). In the literature on biodiversity, the so-called 'rivet hypothesis' (Ehrlich and Ehrlich 1981; Lawton 1993) alleges that:

(...) the functions of species in ecosystems can be analogous to the functions of rivets in an airplane. Both systems can afford continual extraction of its constituent components without experimenting a loss of function. However, after a certain point this capacity is lost and only one additional species extinction (rivet popped) may cause a collapse in the functional properties of the system. ${ }^{2}$

\footnotetext{
${ }^{1}$ For an exhaustive literature survey and appraisal, see Elinor Ostrom (2010)'s lecture, delivered when she received the 2009 Bank of Sweden Prize in Economic Sciences in Memory of Alfred Nobel.

${ }^{2}$ This statement is drawn from Muradian (2001, p. 11)'s benchmark discussion of ecological thresholds.
} 
Secondly, the inherent complexity of ecosystems renders the assessment of discrete thresholds usually uncertain. Continual nutrient loading to a lake, for instance, can cause an abrupt move to a turbid state once a certain nutrient concentration has been reached, but the precise turning point also depends on occasional events like droughts and storms which are essentially random (Bachmann et al. 1999). This illustrates the general fact that ecological data will often be quite noisy, so modelling the mechanisms underlying regime shifts and testing the existence of a threshold can pose real theoretical and statistical challenges. ${ }^{3}$

Most economic analyses of natural commons involving uncertainty, regime shifts and strategic interaction have done so in a public good provision context (see, e.g., Boucher and Bramoullé 2010, and the references therein). Sandler and Sterbenz (1990), then Perrings and Pearce (1994), were the first to deal with a non-excludable but rival good subject to uncertain thresholds. ${ }^{4}$ The former looked at the exploitation of a stock resource, showing that uncertainty about the size of the stock will lead risk-averse firms to reduce their exploitation effort compared to what would happen under certainty. The latter considered the preservation of biodiversity, recommending (in a manner consistent with risk averse behavior) that economic activity be reduced further when the location of an ecological threshold is uncertain. Recently, Bramoullé and Treich (2009) studied the effect of uncertainty in a global commons situation. They show that emissions will be lower under uncertainty than under certainty (because polluters are risk averse), so uncertainty can have a positive effect on welfare. They also point out that agents will cooperate less under uncertainty, as cooperation yields lower rewards. This conclusion provides additional motivation for Dupraz et al. (2009), who explicitly seek means to overcome lack of participation in agri-environmental programs.

These works draw attention to certain exogenous characteristics of agents, such as risk aversion, willingness-to-pay and private information, in driving the outcome. Although these certainly are important items (which we come back to in the closing sections of this paper) in understanding the conservation or depletion of common-

\footnotetext{
${ }^{3}$ For a discussion of this general point, together with an overview and appraisal of available statistical methods, see Andersen et al. (2008).

${ }^{4}$ Experimental game theorists and psychologists have also examined this situation since at least Suleiman and Rapoport (1988)'s article.
} 
pool resources, we will rather emphasize here the role of interaction, endogenous communication and cooperation between agents in the presence of a specific, yet rather plausible, type of uncertainty. This is actually a 'classical topic' in the literature on common-pool resources. It has been extensively examined by means of experiments, notably with games quite similar to the one used in this paper (see, e.g., Budescu et al. 1995). The experimental approach, however, must necessarily assume peculiar utility functions and probability distributions. In particular, multimodal distributions, like the ones introduced here to represent scientific uncertainty about discrete thresholds, were excluded, which rules out multiple equilibria of the 'cautious/dangerous/dreadful' sort we study below.

To be precise, our model amends the well-known Nash demand game (Nash 1950; Malueg 2009), also called the 'Divide-the-dollar game,' by supposing that symmetrically informed players are splitting an uncertain amount (of natural capital) which follows a discrete or multimodal probability distribution. This setting seems to capture a number of stylized situations: in climate change negotiations, for instance, parties normally focus on a finite number of collective targets, such as 550 parts per million ( $\mathrm{ppm})$ of carbon dioxide $\left(\mathrm{CO}_{2}\right)$-equivalent - a politically sensible objective that many think is unlikely to prevent major environmental disruptions, $450 \mathrm{ppm}$ which may limit global warming to a manageable level (thought to be $2^{\circ} \mathrm{C}$ ), or 350 ppm - which some scientists and vulnerable countries regard as the upper bound on emissions that guarantees the preservation of the present biosphere.

In contrast with results in the previous literature, this type of uncertainty does not always lead to lower collective demand for natural capital, even when all agents are risk averse: 'cautious equilibria' - where agents altogether behave as if the worst-case scenario were certain - are found to coexist with 'dangerous equilibria' - where the overall request of ecological services might lead to their collapse - and even 'dreadful equilibria' - where agents collectively claim so much of the resource that no unilateral deviation by one agent can stop its exhaustion. We next bring in the (realistic) possibility that agents communicate and cooperate via group deviations (formally, this means we use the strong and coalition-proof equilibrium concepts developed by Aumann 1959, and Bernheim et al. 1987, respectively). As expected, this readily rules out dreadful equilibria. Somewhat less predictable, however, is the fact that 
dangerous equilibria may be vulnerable to group deviations, while cautious equilibria are not. Actually, dangerous equilibria are not coalition-proof when certain cautious equilibria exist. The upshot is that any cautious equilibrium is efficient and Paretodominates all dangerous equilibria that can be reached from it while increasing every agent's claim on natural capital.

These results are initially derived with only two possible ceilings. We show later on that they are qualitatively robust to the presence of an arbitrary finite number of potential thresholds. They hold as well if uncertainty concerning the location of thresholds is captured by a continuous but multimodal probability distribution. The latter situation may reflect the fact that the models and approaches around which scientists tend to cluster offer different confidence intervals or probability distributions rather than point estimates. Multimodal probability distributions are also likely to emerge in climate change policy discussions (see Jones 2003, and Moss and Schneider 2000), from the aggregation of experts' opinions and the Bayesian updating practiced by the Organization for Economic Cooperation and Development (OECD) and the Intergovernmental Panel on Climate Change (IPCC).

On a policy note, these findings support the creation of institutions and governance mechanisms which enhance communication between users of natural commons: for as long as agents can identify a cautious equilibrium in which they all consume less than in the current state, they should cooperate in moving to this precautionary, yet more efficient, situation. As the derivation of our results will show, however, a lower threshold will invite such a move only if the probability associated with it is sufficiently large; the collective adoption of a costly precautionary stance seems therefore robust to the existence of doomsayers.

The rest of the paper unfolds as follows. The upcoming section lays out the mathematical notation and basic model. Section 3 presents and proves our main propositions. Section 4 checks their robustness and generality. Section 5 discusses some of their policy implications. Section 6 brings some concluding remarks. 


\section{The basic model}

Consider a finite set $N=\{1, \ldots, n\}$ of agents (which can be firms, individuals or countries) who must simultaneously decide how much of a natural capital, measured in positive real numbers, they will claim for themselves. Overall demand is sustainable up to a limit, but scientists disagree on the tipping point beyond which the available resource would collapse to 0 . To keep matters simple (generalizations will be made in Section 4), suppose some experts set the ceiling on total demand at 1 while others deem it to be $a \in(0,1)$. These point estimates are given consensual and commonknowledge probabilities $p$ and $(1-p)$ respectively.

Denote $x_{i}$ an agent $i$ 's claim, demand or request (we use these terms interchangeably throughout the paper) on natural capital, $x=\left(x_{i}\right)_{i \in N}$ a request vector or profile, $X=\sum_{N} x_{i}$ total demand, and $X_{-i}=\sum_{j \neq i} x_{j}$ the sum of all agents' claims except agent $i$ 's. The utility an agent $i$ derives from being delivered her request $x_{i}$ is given by $u_{i}\left(x_{i}\right)$, where the function $u_{i}(\cdot)$ is concave (so agents can be risk averse or risk neutral) and nondecreasing. Reaching this consumption level is of course conditional on total demand not exceeding the ecological threshold; otherwise each agent gets $u_{i}(0)=0$.

Concretely, $x_{i}$ might correspond, for example, to a farming area encroaching on some endangered key species' habitat, a certain flow of wastewater being dumped into a lake, a quantity of fish caught, or some level of carbon dioxide emissions accruing into the atmosphere. This brings agent $i$ a positive utility level $u_{i}\left(x_{i}\right)$, provided an underlying key ecological service - in these cases, land fertility, water purification, livestock renewal, or stable weather, respectively - is maintained. Otherwise, agent $i$ 's utility level drops to 0 .

Agent $i$ 's expected payoff in this amended divide-the-dollar game is now given by

$$
v_{i}\left(x_{i}, X_{-i}\right)=u_{i}\left(x_{i}\right) \mathbb{I}(X \leq a)+p u_{i}\left(x_{i}\right) \mathbb{I}(a<X \leq 1)
$$

where $\mathbb{I}(\cdot)$ indicates whether the condition within parenthesis holds $(=1)$ or not $(=0)$. This completes the description of the model, so we can proceed to the derivation of our main results. 


\section{Main results}

We shall now look first at the Nash equilibria of this simple game. These equilibria will next be put to the test of stronger equilibrium concepts - respectively the notions of strong and coalition-proof Nash equilibria - which allow for group as well as individual deviations.

\subsection{Nash equilibria}

Using the current notation, one can characterize an agent $i$ 's best response strategy as follows.

First, let $X_{-i}>a$, so the other agents are asking for more than the lower resource cap $a$ :

a) If $X_{-i} \leq 1$, then agent $i$ will claim $x_{i}=1-X_{-i}$. She can indeed do no better than request natural capital up to the upper (risky) ceiling 1 , since the remaining agents' overall demand is already higher than the safe threshold $a$.

b) If $X_{-i}>1$, however, agent $i$ can claim any amount $x_{i} \geq 0$, since everybody will end up losing the ecological services anyway.

Next, let $X_{-i} \leq a$, so the other agents are asking for less than the inferior limit $a$ :

a) If $u_{i}\left(a-X_{-i}\right) \geq p u_{i}\left(1-X_{-i}\right)$, then agent $i$ does best by claiming $x_{i}=a-$ $X_{-i}$. Requesting the safe amount $a-X_{-i}$ in this case yields more utility than demanding the best (but risky) alternative $1-X_{-i}$.

b) If $u_{i}\left(a-X_{-i}\right)<p u_{i}\left(1-X_{-i}\right)$, however, agent $i$ should then go for $x_{i}=1-X_{-i}$.

This description of best-response strategies shows that three sorts of Nash equilibria are possible: (1) cautious equilibria, in which agents collectively set total demand at the highest secure level $X=a ;(2)$ dangerous equilibria, where agents altogether request natural capital up to the risky upper ceiling $X=1$ and face a probability $1-p$ of exhausting the resource; and dreadful equilibria, wherein everyone's claim on ecological services is so high (i.e. $X_{-i}>1$ for all $i$ ) that no individual adjustment can avoid their collapse. 
Note that no Nash equilibrium exists in which agents collectively ask for an amount of natural capital lower than $a$ or strictly between $a$ and 1 .

Moreover, cautious, dangerous and dreadful Nash equilibria can coexist, despite the fact that all agents are risk averse. This contrasts with the findings reported so far in the literature (see Bramoullé and Treich 2009, for example). The simultaneous presence of these equilibria is also unlikely to be accidental, as the following simple example suggests.

Example 1. Let there be only two agents, with identical utility function $u_{i}\left(x_{i}\right)=\sqrt{x_{i}}$ for $i=1,2$. Suppose $a=0.8$ and $p=0.8$. The strategy profile $x=(0.5,0.5)$ is a dangerous equilibrium because $v_{i}(0.5,0.5)=0.7 \cdot 0.8=0.56>v_{i}(0.3,0.5)=0.54$ for $i=1,2$. At the same time, the profile $x^{\prime}=(0.4,0.4)$ is a cautious equilibrium, since $v_{i}(0.4,0.4)=0.63>v_{i}(0.6,0.4)=0.77 \cdot 0.8=0.62$ for $i=1,2$; and $x^{\prime \prime}=(1.5,1.5)$ is also clearly an equilibrium, a dreadful one which brings each agent's payoff to $0 .{ }^{5}$

In order to grasp the conditions underlying the existence of each type of Nash equilibria, we need to introduce an extra piece of notation. Let $0 \leq \bar{X}_{i} \leq a$ refer to the cut-off demand level such that

$$
\begin{array}{ll}
u_{i}\left(a-X_{-i}\right)>p u_{i}\left(1-X_{-i}\right) & \text { if } X_{-i}<\bar{X}_{i} \\
u_{i}\left(a-X_{-i}\right)<p u_{i}\left(1-X_{-i}\right) & \text { if } X_{-i}>\bar{X}_{i}
\end{array} .
$$

This allows to make the following preliminary statement.

Lemma 1. For all $i$, there is always a unique cut-off value.

Proof. Let $f_{i}\left(X_{-i}\right) \equiv u_{i}\left(a-X_{-i}\right)-p u_{i}\left(1-X_{-i}\right)$. Clearly, $f_{i}^{\prime}=-u_{i}^{\prime}\left(a-X_{-i}\right)+p u_{i}^{\prime}(1-$ $\left.X_{-i}\right)<0$ since the function $u_{i}$ is concave. When $f_{i}(0)$ is negative or 0 , one can set $\bar{X}_{i}=0$. If $f_{i}(0)$ is positive, the fact that $f_{i}(a)<0$ and $f_{i}($.$) is decreasing and$ continuous entails that there is a unique $\bar{X}_{i}>0$ such that $f_{i}\left(\bar{X}_{i}\right)=0, f_{i}\left(X_{-i}\right)>0$ if $X_{-i}<\bar{X}_{i}$, and $f_{i}\left(X_{-i}\right)<0$ if $X_{-i}>\bar{X}_{i}$.

\footnotetext{
${ }^{5}$ Although we exclude such risk attitudes, note that all three types of equilibria could exist as well with risk-loving agents. To see this, suppose that $i=1,2, u_{i}\left(x_{i}\right)=x_{i}^{2}, p=0.4$ and $a=0.8$. One can check that $x=(0.5,0.5)$ is a dangerous equilibrium, $x^{\prime}=(0.4,0.4)$ is again a cautious one, and $x^{\prime \prime}=(1.5,1.5)$ is a dreadful equilibrium.
} 
The following proposition will now indicate when there always exists at least one cautious or one dangerous equilibrium, and when both types of equilibria actually coexist.

Proposition 1. The game always admits at least one equilibrium which is not dreadful. More precisely,

i) There is a cautious equilibrium if and only if $\sum_{i \in N} \bar{X}_{i} \geq(n-1) a$;

ii) There is a dangerous equilibrium if and only if $\sum_{i \in N} \bar{X}_{i} \leq n-1$;

iii) Both cautious and dangerous equilibria will coexist if and only if

$$
(n-1) a \leq \sum_{i \in N} \bar{X}_{i} \leq n-1 .
$$

Proof. Part (i): By the above description of best-response strategies, a strategy profile $x$ is a cautious equilibrium if and only if

$$
\left\{\begin{array}{l}
X_{-i} \leq \bar{X}_{i} \quad \text { for all } i \in N \\
\sum_{j} x_{j}=a
\end{array}\right.
$$

Using the fact that $X_{-i}=a-x_{i}$ and adding up all the inequalities in (3), we have that $\sum_{i} \bar{X}_{i} \geq(n-1) a$. Conversely, if $\sum_{i} \bar{X}_{i} \geq(n-1) a$, one can always find a vector $x$ which satisfies (3).

Part (ii): Similarly, a strategy profile $x$ is a dangerous equilibrium if and only if

$$
\left\{\begin{array}{l}
X_{-i} \geq \bar{X}_{i} \quad \text { for all } i \in N \\
\sum_{j} x_{j}=1
\end{array}\right.
$$

Using the fact that $X_{-i}=1-x_{i}$ and adding up all the inequalities in (4), we have that $\sum_{i} \bar{X}_{i} \leq n-1$. Conversely, if $\sum_{i} \bar{X}_{i} \leq n-1$, one can always find a vector $x$ which satisfies (4).

Part (iii) follows trivially.

Figure 1 illustrates the sets of equilibria predicted in the proposition, in the twoagent case. These sets depend on the location of the cut-offs $\bar{X}_{i}$, which in turn depends on the lower bound $a$, the probability $p$, and the agents' respective utility functions $u_{i}(\cdot)$. 




Figure 1: The two-agent case.

If agent $i$ becomes more risk averse (so the coefficient of absolute risk aversion $u_{i}^{\prime \prime}(\cdot) / u_{i}^{\prime}(\cdot)$ uniformly increases, say), the cut-off $\bar{X}_{i}$ increases because a secure amount of resources $\left(a-X_{-i}\right)$ now yields relatively more utility than the higher but risky amount $\left(1-X_{-i}\right)$. From proposition 1, one infers that the set of cautious equilibria expands while the set of dangerous equilibria shrinks.

A decrease in the probability $p$ that the actual threshold on ecological services is at 1 instead of $a$ leads to the same conclusion, for $\bar{X}_{i}$ must then go up.

Finally, consider an increase of the lower threshold from level $a$ to level $a^{\prime}$. Since $f_{i}\left(a^{\prime}, p, \bar{X}_{i}\left(a, p, u_{i}\right)\right)>0$, we have that $\bar{X}_{i}\left(a^{\prime}, p, u_{i}\right)>\bar{X}_{i}\left(a, p, u_{i}\right)$. This means that the set of dangerous equilibria gets smaller. However, the set of cautious equilibria might not expand: by proposition 1 , this will happen if and only if $\sum_{N} \bar{X}_{-i}\left(a^{\prime}, p\right)-$ $\sum_{N} \bar{X}_{-i}(a, p)>a^{\prime}-a$.

From these brief remarks about comparative statics, we shall now move on to examine what happens to the above Nash equilibria when group deviations (and not just individual ones) are permitted. 


\subsection{Allowing for communication and cooperation}

Users of common-pool resources can normally communicate, negotiate and act collectively. Standard Nash equilibria a priori discard this behavior, so one may cast doubts about their plausibility in the present context. ${ }^{6}$ A direct consequence of allowing agents to talk to each other and eventually collude could be, for instance, that subgroups may form in which members prefer to jointly move away from some Nash equilibrium. This section will now examine whether the three types of equilibria defined above are immune to such deviations.

Recall that a Nash equilibrium $x$ is strong if there is no alternative strategy profile $x^{\prime} \in \mathbb{R}_{+}^{n}$ such that $v_{i}\left(x^{\prime}\right) \geq v_{i}(x)$ for all agents $i$ belonging to a subcoalition $T \subseteq N$, the inequality being strict for at least one $i$, and $x_{j}^{\prime}=x_{j}$ for all the outsiders $j \in N \backslash T$. The following proposition determines whether dreadful and cautious equilibria are strong in this sense.

Proposition 2. Cautious Nash equilibria are strong, but dreadful Nash equilibria are not.

Proof. From a dreadful equilibrium, any group deviation leading to a cautious or a dangerous strategy profile, be it a deviation by the entire set of players, obviously brings a higher payoff to all agents in the coalition. Hence, dreadful equilibria are not strong Nash equilibria.

The proof that cautious equilibria are strong Nash equilibria proceeds by contradiction. Let $x \in \mathbb{R}_{+}^{n}$ be a cautious Nash equilibrium, and suppose there exists another strategy profile $x^{\prime}$ and a coalition $T \subseteq N$ such that $x_{k}^{\prime}=x_{k}$ for all $k \notin T, v_{i}\left(x^{\prime}\right)>v_{i}(x)$ for some $i \in T$, and $v_{j}\left(x^{\prime}\right) \geq v_{j}(x)$ for all $j \in T$. Since the utility functions $u_{j}$ 's are increasing, it must be the case that $\sum_{j \in T} x_{j}^{\prime}>\sum_{j \in T} x_{j}$ and $x_{j}^{\prime} \geq x_{j}$ for all $j \in T$. Now, consider an agent $j \in T$ such that $X_{-j}^{\prime}>X_{-j}$. For this agent, demanding $x_{j}^{\prime}=a-X_{-j}^{\prime}$ or less leads to a lower payoff than before; her best response must be $x_{j}^{\prime}=1-X_{-j}^{\prime}$. We then have that $v_{j}\left(x_{j}^{\prime}, X_{-j}^{\prime}\right)=v_{j}\left(1-X_{-j}^{\prime}, X_{-j}^{\prime}\right)<v_{j}\left(1-X_{-j}, X_{-j}\right) \leq v_{j}(x)$, where

\footnotetext{
${ }^{6}$ Quoting Ostrom (2010, p. 648), for instance, on why the prisoner's dilemma game might not fully capture what goes on in the sharing of common-pool resources: "Public investigators purposely keep prisoners separated so they cannot communicate. The users of a common-pool resource are not so limited."
} 
the last inequality holds because $x$ is a Nash equilibrium. Agent $j$ is thus worse off under $x^{\prime}$ than under $x$, which contradicts the initial assertion.

This proposition entails that all cautious equilibria are Pareto efficient. Furthermore, any cautious equilibrium Pareto-dominates all dreadful ones. The status of dangerous equilibria is not so clear-cut, however. To analyze it, we shall invoke a weaker equilibrium notion which forbids certain improbable group deviations. Let us call a group deviation self-enforcing if it is not itself exposed to improving subgroup deviations. Following Bernheim et al. (1987), a Nash equilibrium is said to be coalition-proof if no self-enforcing group deviation can deliver an outcome that is Pareto improving. ${ }^{7}$ Clearly, any strong Nash equilibrium (hence any cautious equilibrium, by the last proposition) is coalition-proof. A reconsideration of dangerous equilibria is now at hand.

Proposition 3. Let the strategy profile $x$ be a dangerous equilibrium. If there is a cautious equilibrium $x^{\prime}$ such that, for some subset $T \subseteq N$,

$$
\begin{aligned}
& x_{i}^{\prime}=x_{i}-\alpha_{i} \quad \text { for all } i \in T, \text { and } \\
& x_{i}^{\prime}=x_{i} \quad \text { for all } i \notin T
\end{aligned}
$$

with $\alpha_{i} \geq 0$ for all $i \in T$ and $\sum_{i} \alpha_{i}=1-a$, then $x$ is not coalition-proof.

Proof. Suppose a dangerous equilibrium $x$ is coalition-proof, but that there is a cautious equilibrium $x^{\prime}$ verifying condition (5). For all $i \in T$, we have that

$$
\begin{aligned}
u_{i}\left(x_{i}^{\prime}\right) & \geq p u_{i}\left(x_{i}^{\prime}+1-a\right) \\
& =p u_{i}\left(x_{i}+\sum_{j \neq i} \alpha_{j}\right) \\
& >p u_{i}\left(x_{i}\right) .
\end{aligned}
$$

The first inequality holds because $x^{\prime}$ is itself a Nash equilibrium. The second (strict) inequality follows from the fact that $\sum_{j \neq i} \alpha_{j}>0$, for $\sum_{j \neq i} \alpha_{j}=0$ would mean

\footnotetext{
${ }^{7}$ The formal definition of coalition-proofness (which can be found in Bernheim et al. 1987, or in Moreno and Wooders 1996) is recursive and involves heavy notation that we chose to spare the reader. The application made here of this equilibrium concept is anyhow rather straightforward.
} 
that $x$ is not a Nash equilibrium (since the cautious equilibrium $x^{\prime}$ could then be reached from it through a unilateral move by agent $i$ ). Now, $x^{\prime}$ being a strong Nash equilibrium by proposition 2 , it constitutes a self-enforcing group deviation from $x$. Hence, $x$ cannot be coalition-proof.

Note that condition (5) does not always hold. Suppose, for example, that we have three agents whose preferences are such that $\bar{X}_{1}=\bar{X}_{2}=\bar{X}_{3}=\frac{2 a}{3}$, with $a<\frac{3}{4}$. One can see that $x=\left(0, \frac{1}{2}, \frac{1}{2}\right)$ is a dangerous equilibrium while $x^{\prime}=\left(\frac{a}{3}, \frac{a}{3}, \frac{a}{3}\right)$ is a cautious one. Yet, (5) does not apply to this pair. The important upshot is that coalition-proof dangerous and cautious equilibria can coexist. This also suggests that some dangerous equilibria which are not coalition-proof may not verify condition (5), as the following example shows.

Example 2. Let us add a third identical player to the previous Example 1 where $p=0.8, a=0.8$, and $u_{i} \equiv \sqrt{ }$. Clearly, the request profile $x=\left(\frac{1}{2}, \frac{1}{2}, 0\right)$ is a dangerous equilibrium since $\left(\frac{1}{2}, \frac{1}{2}\right)$ was a dangerous equilibrium in the two-agent game. A profitable deviation for coalition $T=\{1,2\}$ is to demand $(0.4,0.4)$; this deviation is self-enforcing since $(0.4,0.4)$ was a cautious equilibrium in the earlier game. But $x^{\prime}=(0.4,0.4,0)$ is not a cautious equilibrium because the third agent can certainly gain by asking for a share of natural capital larger than zero. Hence, $x$ is not coalition-proof even though no cautious equilibrium verifying expression (5) exists.

This example illustrates the well-known shortcoming of coalition-proofness: a selfenforcing group deviation may be exposed to some outsiders repositioning. More importantly, it also suggests (as Figure 1 somewhat does) that a group deviation from a dangerous to a cautious equilibrium can only occur when claims on natural capital are 'not too asymmetric' across agents. This observation has ramifications for public policy and climate change negotiations that will be briefly discussed in Section 5. Beforehand, we will first check the robustness of the results just shown.

\section{Extensions}

The game considered so far had only two thresholds. This section will now successively contemplate a situation with multiple thresholds and one with a continuum of possible 
thresholds randomly spread according to a multimodal probability distribution. The former case could stem from greater disagreement among experts, the latter from acknowledging measurement errors and aggregating various interval or probabilistic estimates (instead of point estimates) of thresholds. Our analysis will proceed as in the previous section and will produce qualitatively similar results.

\subsection{Multiple thresholds}

Suppose the predicted ecological thresholds, according to a group of experts or models, now belong to a finite set, $A=\left\{a_{1}, \ldots, a_{m}\right\} \subset \mathbb{R}_{+}$. The ecosystem capacity is then either $a_{1}>0$ with probability $p_{1}, a_{2}>a_{1}$ with probability $p_{2}, \ldots$, or $a_{m}>a_{m-1}$ with probability $p_{m}=1-p_{1}-p_{2}-\ldots-p_{m-1}$.

As before, let each agent $i=1, \ldots . n$ claim an amount $x_{i} \geq 0$ of ecological services. If all claims add up to less than or exactly the actual threshold, agent $i$ 's demand, $x_{i}$, is met. On the other hand, if the sum, $X$, of all demands exceeds the true capacity, every agent gets 0 . Let $\bar{p}_{i}=\sum_{j=i}^{m} p_{j}$; agent $i$ 's expected payoff is given by

$$
v_{i}\left(x_{i}, X_{-i}\right)=u\left(x_{i}\right) \mathbb{I}\left(X \leq a_{1}\right)+\ldots+\bar{p}_{m} u_{i}\left(x_{i}\right) \mathbb{I}\left(a_{m-1}<X \leq a_{m}\right)
$$

Proceeding as we did in Section 3 , let $\bar{X}_{i}^{j}$ with $i \in N$ and $j=1, \ldots, m-1$ denote the cut-off values defined as:

$$
\begin{array}{cc}
\bar{p}_{j} u_{i}\left(a_{j}-X_{-i}\right)>\bar{p}_{j+1} u_{i}\left(a_{j+1}-X_{-i}\right) & \text { if } X_{-i}<\bar{X}_{i}^{j} \\
\bar{p}_{j} u_{i}\left(a_{j}-X_{-i}\right)<\bar{p}_{j+1} u_{i}\left(a_{j+1}-X_{-i}\right) & \text { if } X_{-i}>\bar{X}_{i}^{j}
\end{array}
$$

The following statement is a generalization of Lemma 1.

Lemma 2. The cut-offs of each agent $i$ follow the natural order:

$$
0=\bar{X}_{i}^{0} \leq \bar{X}_{i}^{1} \leq \bar{X}_{i}^{2} \leq \ldots \leq \bar{X}_{i}^{m-1} \leq \bar{X}_{i}^{m}=a_{m}
$$

Hence, demanding $x_{i}=a-X_{-i}$ is the best response to $X_{-i} \in\left[\bar{X}_{i}^{k-1}, \bar{X}_{i}^{k}\right]$.

Proof. See the appendix. 
Let us now call cautious a Nash equilibrium strategy profile $x^{*}$ where $X^{*}=\sum_{i=1}^{n} x_{i}^{*}=$ $a_{1}$, h-dangerous a Nash equilibrium in which $X^{*}=a_{h}$, and dreadful a Nash equilibrium where $X^{*}>a_{m}$. Conditions that guarantee the existence of these equilibria are given below.

Proposition 4. The game always admits at least one non-dreadful equilibrium. Furthermore,

1) A cautious equilibrium exists if and only if $\sum_{i \in N} \bar{X}_{i}^{1} \geq(n-1) a_{1}$;

2) For any $h \in\{2, \ldots, m\}$, an $h$-dangerous equilibrium exists if and only if

$$
\sum_{i \in N} \bar{X}_{i}^{k-1} \leq(n-1) a_{k} \leq \sum_{i \in N} \bar{X}_{i}^{k}
$$

This proposition implies that cautious and all $h$-dangerous equilibria may coexist. The underlying argument matches the one used in the proof of proposition 1.

The possibility of group deviations would again make dreadful equilibria and certain dangerous equilibria untenable. The following statements are straightforward extensions of propositions 2 and 3 .

Proposition 5. Cautious Nash equilibria are strong; dreadful Nash equilibria are not.

Proposition 6. Let a strategy profile $x$ be an h-dangerous equilibrium. If there is an $h^{\prime}$-dangerous equilibrium $x^{\prime}, h^{\prime}<h$, such that for a subgroup $T \subseteq N$,

$$
\begin{aligned}
& x_{i}^{\prime}=x_{i}-\alpha_{i} \quad \text { for all } i \in T \text {, and } \\
& x_{i}^{\prime}=x_{i} \quad \text { for all } i \notin T
\end{aligned}
$$

with $\alpha_{i} \geq 0$ for all $i \in T$ and $\sum_{i} \alpha_{i}=1-a_{h^{\prime}}$, then $x$ is not coalition-proof.

More clearly than its counterpart in section 3, the latter proposition conveys a notion of gradualism (which may matter, for instance, in international climate policy negotiations). The $h^{\prime}$-equilibrium that a group of agents would prefer to the riskier $h$-equilibrium may not itself be coalition-proof. In this case, one can imagine that another deviation to an even safer $h^{\prime \prime}$-equilibrium might occur, and so on, until a cautious equilibrium is reached. 
We shall now show that these results do not depend (qualitatively, at least) on having the natural threshold take only a finite number of possible values.

\subsection{Continuous (multimodal) distributions of thresholds}

Consider now a situation where the ceiling on ecological services $r$ is located according to a common-knowledge distribution function $F(\cdot)$ with density $f(\cdot)$ such that $F(0)=$ 0 . We view $F(\cdot)$ as an aggregate of various interval or probabilistic estimates of an ecological threshold and assume it is multimodal.

Again, let each agent $i=1, \ldots n$ make a claim $x_{i} \geq 0$ on natural capital. If the sum $X$ of individual demands amounts to no more than $r$, all requests $x_{i}$ are satisfied; otherwise, everybody gets 0 . The probability of not exceeding the threshold being given by $1-F(X)$, agent $i$ 's expected payoff is then

$$
v_{i}\left(x_{i}, X_{-i}\right)=u_{i}\left(x_{i}\right)\left(1-F\left(x_{i}+X_{-i}\right)\right)
$$

Notice first that this payoff function can have several local maxima. The first-order necessary condition for maximizing agent $i$ 's payoff is given by

$$
\frac{u_{i}^{\prime}\left(x_{i}\right)}{u_{i}\left(x_{i}\right)}=\frac{f\left(x_{i}+X_{-i}\right)}{1-F\left(x_{i}+X_{-i}\right)}
$$

The left-hand side of this expression increases with $x_{i}$. The right-hand side is the inverse of the so-called hazard rate. The literature on mechanism design (see, e.g., Bulow and Roberts 1989; Levin 1997) usually assumes the latter to be monotonically decreasing. Here, the fact $F(\cdot)$ is multimodal may cause it to be non-monotonic, since between two sufficiently far off predictions the density $f(\cdot)$ will first decline and then grow sharply. This may indeed create multiple local maxima.

In this context, let us call a Nash equilibrium cautious (dangerous) when total demand is at the lowest (highest) level. Both types of equilibria may coexist, even in this continuous framework, as the following examples illustrate. A notable difference with the discrete case is that there can be only one Nash equilibrium of a kind.

Example 3. Suppose there are three agents with identical utility function $u_{i}\left(x_{i}\right)=$ $\sqrt{x_{i}}, i=1,2,3$. Let the distribution of thresholds $F(\cdot)$ be the weighted sum of two 
different expert assessments: the first one is a normal distribution with mean equal to 8 and variance equal to 1 , the second is also a normal distribution with mean 18 and variance 1 . The respective weights put on the former and the latter are 0.8 and 0.2. Two symmetric Nash equilibria will coexist in this case: one (the cautious equilibrium) in which an agent claims an amount 2.325 of natural capital, and the other (the dangerous equilibrium) in which an agent requests 5.421.

Example 4. Using the same bimodal distribution as in example 3, suppose there are now three risk-neutral agents with identical utility function $u_{i}\left(x_{i}\right)=\lambda * x_{i}, i=1,2,3$. Again, two symmetric equilibria will coexist in this game: in the cautious equilibrium an agent claims an amount 2.47 of ecological services, in the dangerous equilibrium an agent demands 5.55.

These examples also reveal that, contrary to what happens when there is a finite number of possible thresholds, overall demand for natural capital changes with the agents' degree of risk aversion. In both the cautious and the dangerous equilibrium, claims are actually lower (as expected) when agents are more risk averse.

As the next proposition shows, finally, the cautious equilibrium in this continuous setting is still a strong Nash equilibrium; it dominates any dangerous equilibrium in which every agent makes a bigger claim.

Proposition 7. The cautious equilibrium is a strong Nash equilibrium. It Paretodominates any dangerous equilibrium in which everyone asks for more natural capital.

Proof. The argument for the first part mimics that for proposition 2. Denote by $x$ the cautious equilibrium of the game. Consider another equilibrium, $x^{\prime}$, to be the result of a profitable deviation by coalition $T \subseteq N$. Clearly $|T| \geq 2$, or $x$ would not be a Nash equilibrium of the game. Also, it must be that $X^{\prime} \neq X$. Suppose $X^{\prime}>X$, and consider an agent $j \in T$ such that $X_{-j}^{\prime}>X_{-j}$. Such an agent exist because $|T| \geq 2$. Denote by $x_{j}^{*}\left(X_{-j}^{\prime}\right)$ agent $j$ 's best response to all other agents collectively demanding 
$X_{-j}^{\prime}$. By the monotonicity of $u_{j}$, it must be that

$$
\begin{aligned}
v_{j}\left(x_{j}^{\prime}, X_{-j}^{\prime}\right) & \leq v_{j}\left(x_{j}^{*}\left(X_{-j}^{\prime}\right), X_{-j}^{\prime}\right) \\
& =u_{j}\left(x_{j}^{*}\left(X_{-j}^{\prime}\right)\right) \times\left(1-F\left(x_{j}^{*}\left(X_{-j}^{\prime}\right)+X_{-j}^{\prime}\right)\right) \\
& <u_{j}\left(x_{j}^{*}\left(X_{-j}^{\prime}\right)+X_{-j}^{\prime}-X_{-j}\right) \times\left(1-F\left(x_{j}^{*}\left(X_{-j}^{\prime}\right)+X_{-j}^{\prime}\right)\right) \\
& =v_{j}\left(x_{j}^{*}\left(X_{-j}^{\prime}\right)+X_{-j}^{\prime}-X_{-j}, X_{-j}\right) \\
& \leq v_{j}\left(x_{j}, X_{-j}\right)
\end{aligned}
$$

with the last inequality coming from the fact that $x$ is a Nash equilibrium of the game. A cautious equilibrium being a Nash equilibrium with the lowest total demand by definition, the case $X^{\prime}<X$ cannot happen.

To prove the second part, denote by $x$ the cautious equilibrium of the game and by $x^{\prime}$ a dangerous equilibrium such that $X_{-i}^{\prime} \geq X_{-i}$ for all $i \in N$. From the definition of an equilibrium, we know that for all $i$

$$
v_{i}\left(x_{i}, X_{-i}\right) \geq v_{i}\left(x_{i}^{\prime}, X_{-i}\right) .
$$

Furthermore, $v_{i}$ is non-increasing in its second argument, because a larger value implies smaller odds of obtaining a positive payoff. Therefore,

$$
v_{i}\left(x_{i}^{\prime}, X_{-i}\right) \geq v_{i}\left(x_{i}^{\prime}, X_{-i}^{\prime}\right) .
$$

Combining the last two expressions hands the result.

This completes our verification that the results shown in Section 3 are qualitatively robust. We shall now explore some of their main ramifications for public policy.

\section{Some policy implications}

The existence of an ecological threshold, and the fact that its exact level is uncertain, raise specific policy issues. An important one is whether to then adopt precautionary measures. The feasibility and social desirability of such actions have been widely debated in policy circles and remain an active research topic (see, e.g., 
Wiener 2010, Barrieu and Sinclair-Desgagné 2006, and the references therein). In the present context, choosing a precautionary stance would mean to avoid claiming natural capital beyond the lowest possible threshold, in order to safeguard those vital and irreplaceable ecological services. Contrary to the current literature, which assumes a benevolent planner or representative agent with the proper kind of risk or ambiguity aversion, proposition 1 above shows that this outcome can actually be achieved in a decentralized fashion: it corresponds to what we called a 'cautious' equilibrium.

Propositions 2 and 3 entail, moreover, that precautionary outcomes would arise rather naturally as a result of fostering communication and collaboration between agents, since cautious equilibria are robust to group deviations (hence are also Pareto optimal) while dreadful equilibria are not and dangerous equilibria may not.

There is, however, one caveat to proposition 3 which is worth mentioning. In that proposition, the coalition which departs from the dangerous equilibrium, thereby implementing a precautionary outcome, bears all the cost while outsiders retain their initial claim (which will moreover materialize with certainty). This could create an incentive for agents to stick to their original request and free-ride on deviating coalitions, so dangerous equilibria might not be that vulnerable after all. One way around this situation is to seek a grand coalition in which everybody settles for less natural capital. This is the approach chosen notably in the post-Kyoto negotiations over national reductions in carbon dioxide emissions. It might be the only sensible one when agents are fairly similar in their respective preferences and requests, but the effort then spent in drawing everybody together might be daunting. Alternatively, if there is 'enough asymmetry' between agents at some dangerous equilibrium outcome $x$ (but not too much, in light of the discussion that follows example 2 above), a coalition of the willing, ready to implement a precautionary outcome, might come about as follows. Using proposition 3's notation, let $x_{i}-\alpha_{i}^{C E}$ be the certainty equivalent for agent $i$, so $\alpha_{i}^{C E}$ is the positive quantity such that $u_{i}\left(x_{i}-\alpha_{i}^{C E}\right)=p u_{i}\left(x_{i}\right)$. The idea is to target the agents with the largest $\alpha_{i}^{C E}$, in order to subsequently have them give up an amount of natural capital $\alpha_{i}=\alpha_{i}^{C E}-\varepsilon$ where $\varepsilon$ is a small positive number. These agents will typically combine relatively high risk aversion with large claims on ecological services. Suppose they form a strict subgroup $T \subseteq N$ such that $\sum_{i \in T} \alpha_{i}^{C E}>1-a$. If, in addition, the magnitude of their potential to sacrifice 
would make their individual participation essential in any successful group deviation, then they might well choose to collectively move ahead in deviating to a cautious equilibrium.

\section{Concluding remarks}

This paper has analyzed the behavior of rational agents sharing a non-excludable ecosystem that may not deliver some key services beyond an uncertain ecological threshold. We modelled this situation as a version of the well-known 'Divide-thedollar' game in which the amount to be split follows a discrete probability distribution. This brought two new insights for the economics of common-pool resources. First, strategic interaction can have much more impact on the outcome than individual features: whatever the agents' respective degree of risk aversion, for instance, 'cautious' equilibria - where agents altogether behave as if the lowest (and safest) threshold were certain - were found to coexist with 'dangerous' equilibria - where the agents' request of ecological services up to a higher threshold might lead to an ecosystem breakdown

- and even 'dreadful' equilibria - where so much natural capital is claimed collectively that no single player's actions can prevent its exhaustion. Second, allowing agents to cooperate and form deviating coalitions would eliminate all dreadful equilibria and several dangerous equilibria, while cautious equilibria were shown to be robust to such deviations. These results support the emphasis currently put by the common-pool resources literature on social capital, governance and institutions. The latter also captures the recurrent empirical observation that some resource-sharing communities were able to avoid a tragedy of the commons through communication and cooperation (Ostrom 1999); its derivation indicates, moreover, which dangerous equilibria will be discarded and which situation a successful deviating coalition will then prefer.

The above analysis and conclusions may first apply to other contexts as well. Abusing natural capital might trigger severe social unrest instead or in addition of a loss of ecological services (as in Diamond 2005's tale of the Mayas collapse through drought and warfare), for instance, or the agents' actions might directly encroach on social (rather than natural) capital until some uncertain borderline is crossed and civil war erupts (as in André and Platteau 1998's account of the rising tensions in 
social and family relations that increasing land scarcity engendered in the eighties in Rwanda, which partly paved the way to the bloody civil war that broke out in 1994.) Another immediate step from this paper, considering the relative simplicity of the present game, would be to check whether the above results hold as well in the laboratory. In such a setting, we expect the presence of focal points, or the subjects' usual preference for equality and fairness (as reported in Ostrom 2000), to then reduce the initial sets of equilibria but also affect the extent to which deviating coalitions may form. Other theoretical insights could additionally be obtained by relaxing some informational assumptions. For example, agents might be allowed to hold different beliefs about the location and distribution of thresholds or have access (at some cost) to a privately observable warning signal (of the type discussed, say, in Scheffer et al. 2009). The issue of asymmetric information has already been considered in the common-pool resource literature (see, e.g., Lindahl and Johannesson 2009, and the references therein), but not with various discrete thresholds or when the distribution of thresholds is multimodal, as above. Finally, one should examine rigorously how agents communicate and coordinate with each other. To be sure, for the above equilibrium outcomes to locate where potential thresholds precisely lie, some means of coordination must exist (particularly in large-scale natural commons, such as open seas or the earth atmosphere). It would be worthwhile to investigate what they are, and to explore when and how they can support cooperation. 


\section{References}

[1] Andersen, Tom, Jacob Carstensen, Emilio Hernandez-Garcia and Carlos M. Duarte. 2008. Ecological thresholds and regime shifts: approaches to identification, Trends in Ecology and Evolution 24, 49-57.

[2] André, Catherine, and Jean-Philippe Platteau. 1998. Land relations under unbearable stress: Rwanda caught in the Malthusian trap, Journal of Economic Behavior and Organization 34, 1-47.

[3] Aumann, Robert J. 1959. Acceptable points in general cooperative n-person games, in Contributions to the Theory of Games IV, Annals of Mathematical Studies, vol. 40 (ed. by R. D. Luce and A. W. Tucker), Princeton University Press, p. 287-324.

[4] Bachmann, R., M. Hoyer and D. Canfield. 1999. The restoration of Lake Apopka in relation to alternative stable states, Hydrobiologia 394, 219-232.

[5] Barrieu, Pauline and Bernard Sinclair-Desgagné. 2006. On precautionary policies, Management Science 52, 1145-1154.

[6] Bernheim, Douglas, Bezalel Peleg and Michael D. Whinston. 1987. Coalitionproof Nash equilibria: I concepts, Journal of Economic Theory 42, 1-12.

[7] Boucher, Vincent and Yann Bramoullé. 2010. Providing global public goods under uncertainty, Journal of Public Economics 94, 591-603.

[8] Bramoullé, Yann and Nicolas Treich. 2009. Can uncertainty alleviate the commons problem?, Journal of the European Economic Association 7, 1042-1067.

[9] Brozović, Nicholas and Wolfram Schlenker. 2010. Optimal management of an ecosystem with an unknown threshold, Mimeo, University of Illinois.

[10] Budescu, David V., Amnon Rapoport and Ramzi Suleiman. 1995. Common pool resource dilemmas under uncertainty: qualitative tests of equilibrium solutions, Games and Economic Behavior 10, 171-201. 
[11] Bulow, Jeremy and John Roberts. 1989. The Simple Economics of Optimal Auctions, The Journal of Poilitical Economy, 97, 1060-1090.

[12] Diamond, Jared. 2005. Collapse - How Societies Choose to Fail or Succeed. London UK, Penguin Books.

[13] Dupraz, Pierre, Karine Latouche and Nadine Turpin. 2009. Threshold effect and coordination of agri-environmental efforts, Journal of environmental planning and management 52, 613-630.

[14] Ehrlich, P. and A. Ehrlich. 1981. Extinction: The Causes and Consequences of the Disappearance of Species. Ballantine Books, New York.

[15] Jones, R. 2003. Managing climate change risks, paper delivered at the OECD Workshop on the Benefits of Climate Policy: Improving Information for Policymakers, Paris.

[16] Lawton, J. 1993. The role of species in ecosystems: aspects of ecological complexity and biological diversity, in Biodiversity: An Ecological Perspective (A. D. Schulze and H. Mooney, eds.). Springer, Germany.

[17] Levin, Jonathan. 1997. An Optimal Auction for Complements, Games and Economic Behavior 18, 176-192.

[18] Lindahl, Therese and Magnus Johannesson. 2009. Bargaining over a common good with private information, Scandinavian Journal of Economics 111(3), 547565 .

[19] Malueg, David, 2010. Mixed-strategy equilibra in the Nash demand game, Economic Theory, DOI 10.1007/s00199-009-0478-5

[20] Moss, R. H. and S. H. Schneider. 2000. Uncertainties in the IPCC TAR: Recommendations to lead authors for more consistent assessment and reporting, in Third Assessment Report Guidance Papers (R. Pachauri, T. Taniguchi and K. Tanaka, eds.), World Meteorological Organization, Geneva. 
[21] Muradian, Roldan, 2001. Ecological thresholds: a survey, Ecological Economics $38,7-24$.

[22] Nash, J.F., 1950. The bargaining problem. Econometrica 18, 155-162.

[23] Ostrom, Elinor. 2010. Beyond markets and states: polycentric governance of complex economic systems, American Economic Review 100, 641-672.

[24] Ostrom, Elinor. 1999. Coping with tragedies of the commons, Annual Review of Political Science 2, 493-535.

[25] Perrings, Charles and David Pearce. 1994. Thresholds effects and incentives for the conservation of biodiversity, Environmental and Resource Economics 4, 1328.

[26] Sandler, Todd and Frederic P. Sterbenz. 1990. Harvest uncertainty and the Tragedy of the Commons, Journal of Environmental Economics and Management 18, 155-67.

[27] Scheffer, M., J. Bascompte, W. A. Brock, V. Brovkin, S. R. Carpenter, V. Dakos, H. Held, E. H. van Nes, M. Rietkerk, and G. Sugihara. 2009. Early-warning signals for critical transitions, Nature 461(3), 53-59.

[28] Scheffer, M., S. R. Carpenter, J. A. Foley, C. Folke, and B. Walker. 2001. Catastrophic shifts in ecosystems, Nature 413, 591-596.

[29] Suleiman, Ramzi and Amnon Rapoport. 1988. Environmental and social uncertainty in single-trial resource dilemmas, Acta Psychologica 68, 99-112

[30] Wiener, Jonathan. 2010. The Reality of Precaution. Lavoisier Press, Paris.

\section{A Appendix}

\section{A.1 Proof of Lemma 2}

Let $k \in 1, \ldots, m$, we start by proving that the set $A_{k}:=\left\{y \in \mathbb{R}_{+} \mid \bar{p}_{k} u_{i}\left(a_{k}-y\right)>\right.$ $\left.\max _{j \neq k} \bar{p}_{j} u_{i}\left(\left(a_{j}-y\right)_{+}\right)\right\}$is convex subset of $\left[0, a_{k}[\right.$. This follows from the concavity of 
$u_{i}$ and the fact that the sequence of $\bar{p}_{j}$ 's is decreasing:

i) First, notice that $A_{k} \subseteq\left[0, a_{k}\left[\right.\right.$, because $a_{k}-X_{-i}>0$ on this set only, and agents are not allowed to demand negative amounts of ecological services.

ii) For all $j>k$, the expression $\bar{p}_{k} u_{i}\left(a_{k}-y\right)-\bar{p}_{j} u_{i}\left(\left(a_{j}-y\right)_{+}\right)$is decreasing in $y$ on $\left[0, a_{k}\right]$. Therefore $\left\{y \in\left[0, a_{k}\right] \mid \bar{p}_{k} u_{i}\left(a_{k}-y\right) \geq \bar{p}_{j} u_{i}\left(a_{j}-y\right)\right\}$ is convex.

iii) For all $j<k$, the expression $\bar{p}_{k} u_{i}\left(\left(a_{k}-y\right)_{+}\right)-\bar{p}_{j} u_{i}\left(a_{j}-y\right)$ is increasing on $\left[0, a_{j}\right]$. Moreover, $\bar{p}_{k} u_{i}\left(a_{k}-y\right)>\bar{p}_{j} u_{i}\left(\left(a_{j}-y\right)_{+}\right)=0$ on $\left[a_{j}, a_{k}\right]$. Hence, $\{y \in$ $\left.\left[0, a_{k}\right] \mid \bar{p}_{k} u_{i}\left(a_{k}-y\right) \geq \bar{p}_{j} u_{i}\left(a_{j}-y\right)\right\}$ is also convex.

It follows from the convexity of the intersection of convex sets that the set $A_{k}$ is convex, for all $k$. Moreover, point iii) implies that $A_{j} \leq A_{k}$ for all $j<k$, so that the $A_{k}$ 's are in the natural order.

Finally, denoting $\bar{X}_{i}^{k-1}$ and $\bar{X}_{i}^{k+1}$ the lower and upper bounds of the set $A_{k}$, respectively, yields the result. 